\title{
Adaptation algorithms of mathematical educational content in e-learning courses
}

\author{
Yulia Vainshteina ${ }^{1, *}$, Victoria Shershneva ${ }^{1}$, Roman Esin $^{1}$, Gennady Tsibulsky ${ }^{1}$, and \\ Konstantin Safonov ${ }^{2}$ \\ ${ }^{1}$ Siberian Federal University, Kirenskogo st., 26, Krasnoyarsk, 660074, Russia \\ ${ }^{2}$ Reshetnev University, Krasnoyarskii Rabochii av., 31, Krasnoyarsk, 660037, Russia
}

\begin{abstract}
This article is devoted to the development of algorithms for the adaptation of mathematical educational content and their implementation in the e-learning system. The adaptation of a mathematical content consists in the selection of an optimal edition of the material that best suits the individual characteristics of the student. The novelty of the work is the organization of the adaptation algorithms of the educational content in a three-level system: "introductory content adaptation" (an adaptation of the content of introductory materials of the discipline based on the initial level of students) - "current adaptation of the content" (an adaptation of the mathematical content based on current effective actions of students in the electronic course) - "appraisal-correcting adaptation" (an adaptation of normative parameters of the level of assimilation of materials, taking into account the results achieved by students). The introduction of the proposed three-stage system makes it possible to implement in individual educational trajectories and to form for each student a personal space of mathematical educational content that adapts to its level of mastering the material, which helps to improve the quality of instruction in mathematical disciplines. An approbation of the proposed algorithms was carried out in the adaptive e-course in discrete mathematics, implemented at Siberian Federal University in Russia.

Keywords: Adaptive e-learning cource, teaching mathematics, adaptation of mathematical content, adaptive E-Learning, personalized education, individual educational trajectory, LMS Moodle
\end{abstract}

\section{Introduction}

New educational technologies are developing in the world education system, including the individualization of the educational process in an electronic environment is becoming increasingly widespread. Contemporary trends in the development of training in the electronic environment determine the need to use adaptive electronic educational courses, the experience of development and implementation of which in higher education is currently very limited. Adaptive learning courses in the electronic environment are

* Corresponding author: YWeinstein@sfu-kras.ru 
understood as electronic learning courses that provide the student with a personal educational space filled with mathematical content that adapts to his individual characteristics, providing the necessary information [1]. As individual characteristics of a student, the level of mastering the material in the discipline and the activity in the e-course are considered.

The range of functional tasks solved with the help of adaptive educational courses implemented in e-learning systems is extremely wide, ranging from the organization of independent work of students and distance learning to the development of professional competencies in the conditions of the modern trend "Life Long Learning".

The key tasks in the development of adaptive learning courses in mathematical disciplines are: structuring of educational mathematical content, building the normative model of a learner, and elaboration of algorithms and methods of an automatic navigation for the participants of the educational process in the e-learning system.

The review of research directions in the development and implementation of adaptive educational courses and environments has made it possible to single out the following main directions: designing individual educational trajectories [2-4], searching for approaches to modeling subject areas of disciplines [5-7], the formation of models of learners behaviors [8-11] and the development of adaptation algorithms in learning systems and environments [12-17]. Of course, to elaborate adaptive learning courses in mathematical disciplines with a wide range of functionalities, it is advisable to integrate the results of research in all these directions.

The article is devoted to the development of algorithms for the adaptation of mathematical educational content and their implementation in the e-learning system. A feature of the proposed algorithms is the possibility of its application in an adaptive elearning course (AELC), implemented in learning management systems, including on the platform Learning Management System (LMS) Moodle.

The novelty of the work is that algorithms for adaptation of the educational content are developed within a three-level system: «introductory adaptation of content» (adaptation of the content of the introductory material of discipline on the basis of entry-level students) «the current adaptation of content» (adaptation of mathematical content based on current productive activities students in adaptive e-course) - «appraisal-correcting adaptation» (adaptation of the normative parameters of the level of assimilation of materials, taking into account the student's learning outcomes).

\section{Adaptation algorithms of educational content}

The proposed algorithms for adaptation of mathematical educational content in adaptive ecourses have been tested in the development of AELC in the virtual learning environment LMS Moodle. The wide opportunities for the management of educational activity in the electronic environment LMS Moodle make it one of the most common in the development of AELC in Russian and foreign higher education institution [18]. The general structure of the three-level system of adaptation of educational content in the AELC and the implementation of individual educational trajectories by forming a personal space for study materials for each student is shown in Fig. 1.

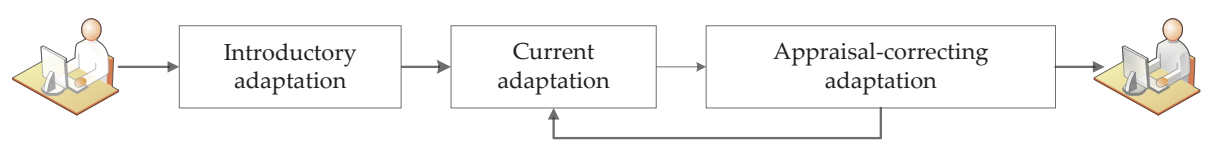

Fig. 1. The system of the educational content adaptation in AELC. 
At the initial stage of the training the introductory adaptation of the content to the individual characteristics of a student is carried out; the enlarged algorithm for this adaptation is shown in Fig. 2.

The algorithm at the introductory adaptation stage includes an assessment of the initial level of a student preparation, identification of gaps in the necessary (basic) mathematical knowledge that interferes with the assimilation of new material in the mathematical discipline. Based on the assessment of the level of initial training, it is recommended to conditionally divide all students into three groups: "low", "medium" and "high" level of training. Students of groups with "low" and "medium" levels are individually recommended additional corrective materials that allow them to fill the missing mathematical knowledge and in the future to quickly learn the material. Thus, at this stage, an individual educational trajectory begins to be built for each student, and by the beginning of the study of the basic material in the adaptive e-course in mathematics everyone reaches a level of preparation that is sufficient for further development of the course.

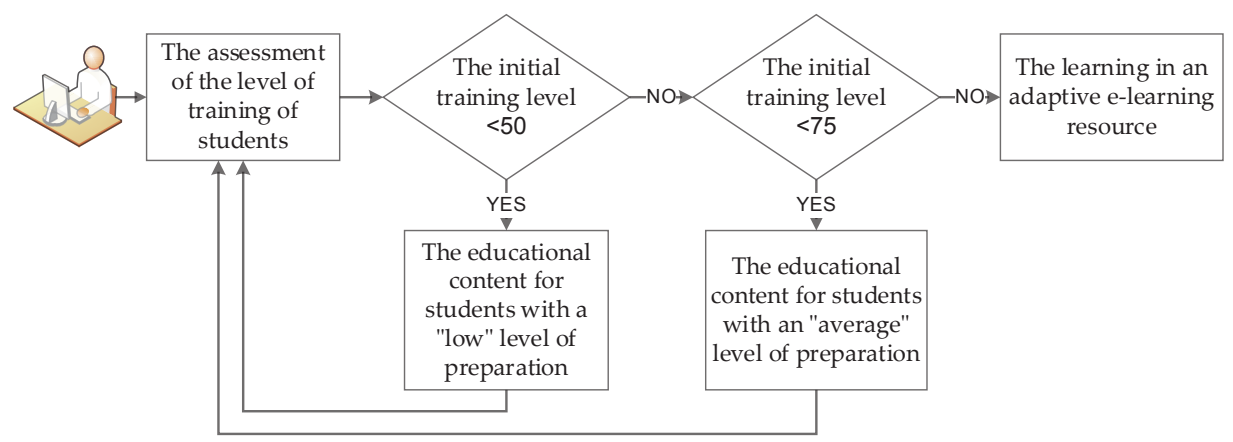

Fig. 2. The algorithm of introductory adaptation in AELC.

A feature of the current adaptation stage is the opportunity to increase the level of mastering of the material by providing the student with the content of the discipline in the edition corresponding to the current level of mastering of the material (the level of mathematical competence formation).

A structuring of the educational content of AELC in mathematical disciplines is proposed to be done by singling out the minimal theoretical units representing the semantically complete microportions of the educational material called terms, and determining the sequence of their study. Principles of the allocation of terms based on the logical and epistemological analysis, proposed by E.K. Voishvillo, are detailed in the work [19].

Based on educational practice, when presenting material in AELC, it often becomes necessary to present it in various editions. This is especially actually for mathematical content in connection with the high level of abstraction and the complexity of the perception of mathematical disciplines. So, it is proposed to use at least three editions of the presentation for each term of mathematical content. In our opinion, the editions of the terms of educational content must be based on the levels of mathematics competence. We have highlighted the following three levels: the level of reproduction, the level of establishing links and dependencies, and the level of reasoning.

The presentation of the term of mathematical content in the first level (reproduction) edition is constructed in such a way that it gives an idea of the application of facts and standard methods in a familiar situation, acquaints with mathematical objects and their properties, standard expressions and formulas, demonstrates the implementation of standard 
procedures and known algorithms, and also the direct execution of calculations and transformations.

The presentation of the term of mathematical content in the edition of the next level (establishment of links and dependencies) contains, along with the material of the first-level term, material demonstrating examples of establishing relationships and dependencies between data in the condition of a task, as well as approaches to analyzing the conditions of tasks and methods of integrating mathematical methods and knowledge in their solution, as well as examples of solving mathematical problems that, although not quite typical, are still familiar to students or to a small extent go beyond the known.

The presentation of the term of mathematical content in the third level (reasoning) edition includes materials aimed at developing skills of independence and initiative, including a problem approach to the presentation of material, encourages students to independently formulate problem solving algorithms, integrate knowledge of the entire course, substantiate and interpret the results.

The proposed features of editions of educational content reflect the specifics of the formation of mathematical competence.

The students get an opportunity to better understand complex theoretical material due to the variable representation of the content of the mathematical term corresponding to different editions. A fragment of the enlarged algorithm of the current adaptation of an educational content, allowing one to switch between its editions E1.1., E1.2, E1.3, depending on the level of mastering the material of the current term 1, then choose one of the versions E2.1., E2.2, E2.3 of the next term 2, and if necessary switch between them, is shown in Fig. 3.

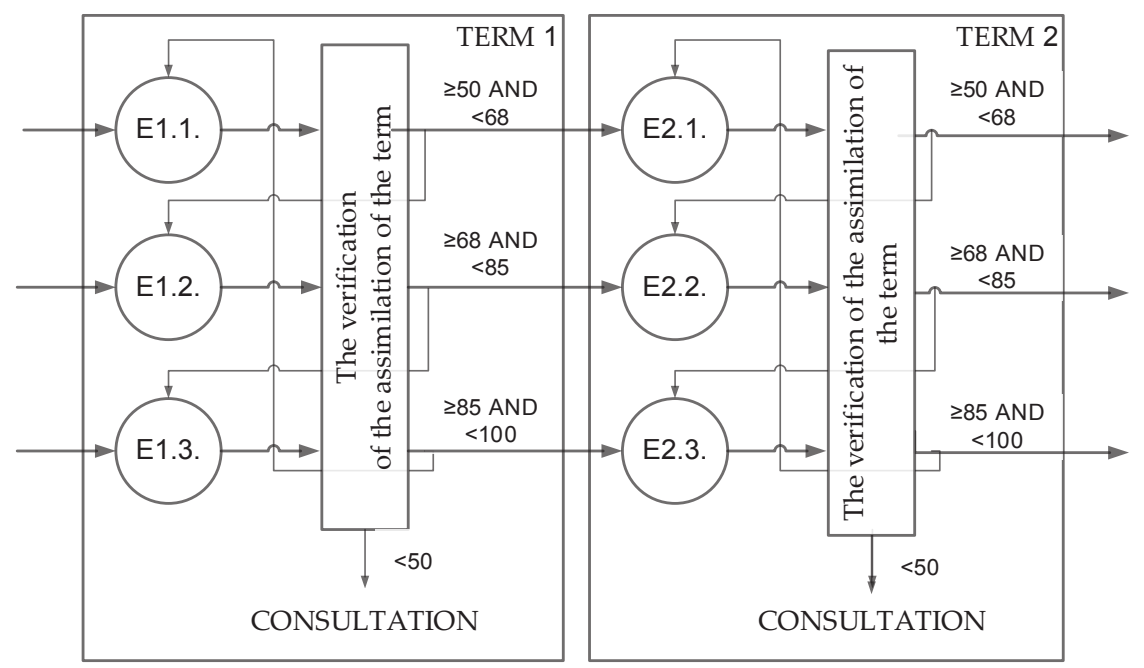

Fig. 3. The algorithm of switching between editions of the term in the process of the current adaptation.

This algorithm allows one to build multiple trajectories with the possibility of relearning the material in various editorial versions of the presentation. The rules for the selection of educational content, included in the model of management of the learning process, allow students to provide optimal editions of the studied terms on the basis of individual characteristics of students. Effective actions of students initiate an automated transition to the study of the materials of the next term or the repetition of materials of the current term in the wording corresponding to the achieved level. 
The process of verifying the assimilation of educational material for each term is realized in the triad of "knowledge-skill-possession." Complex monitoring of the presented triad makes it possible to assess the level of mathematics competence formation expressed in the ability to apply knowledge of mathematical disciplines in professional activity [20].

The purpose of the appraisal-correcting adaptation is the assessment and adjustment of the normative values of the level of assimilation of AELC training materials, which are the parameters of the transition between the editions of materials and the terms of educational content. Initially, the normative values are established on the basis of expert teaching evaluation. Further, based on the results of AELC assimilation by students, the initial normative values are adjusted, based on the average values for the group of students, Fig. 4.

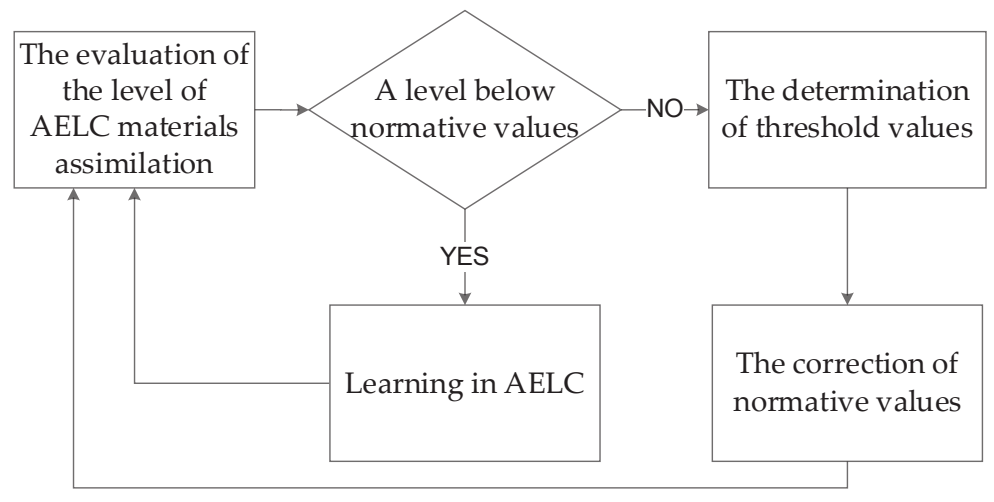

Fig. 4. The algorithm of appraisal-correcting adaptation.

The software implementation of the proposed adaptation scheme was carried out in LMS Moodle by imposing restrictions on the training elements of the electronic course: tracking the execution of the element - studying a determined edition of the term or passing through a checkpoint and monitoring the level of assimilation of the term. The adjustment of AELC elements on the basis of the proposed algorithms makes it possible to realize the variability of the presentation of educational information within the framework of a single e-course, forming an individual profile for the representation of mathematical content for each student.

\section{Results}

An approbation of the algorithms proposed in this report was carried out in AELC in the discipline "Discrete Mathematics" at the Siberian Federal University in Russia. 121 students of the experimental group and 119 students of the control group participated in the experiment. As a result of the final control over the assimilation of the materials of AELC in discrete mathematics, the results of which are shown in Fig. 5, the effectiveness of the experimental group was on average $14 \%$ higher than in the control group. 


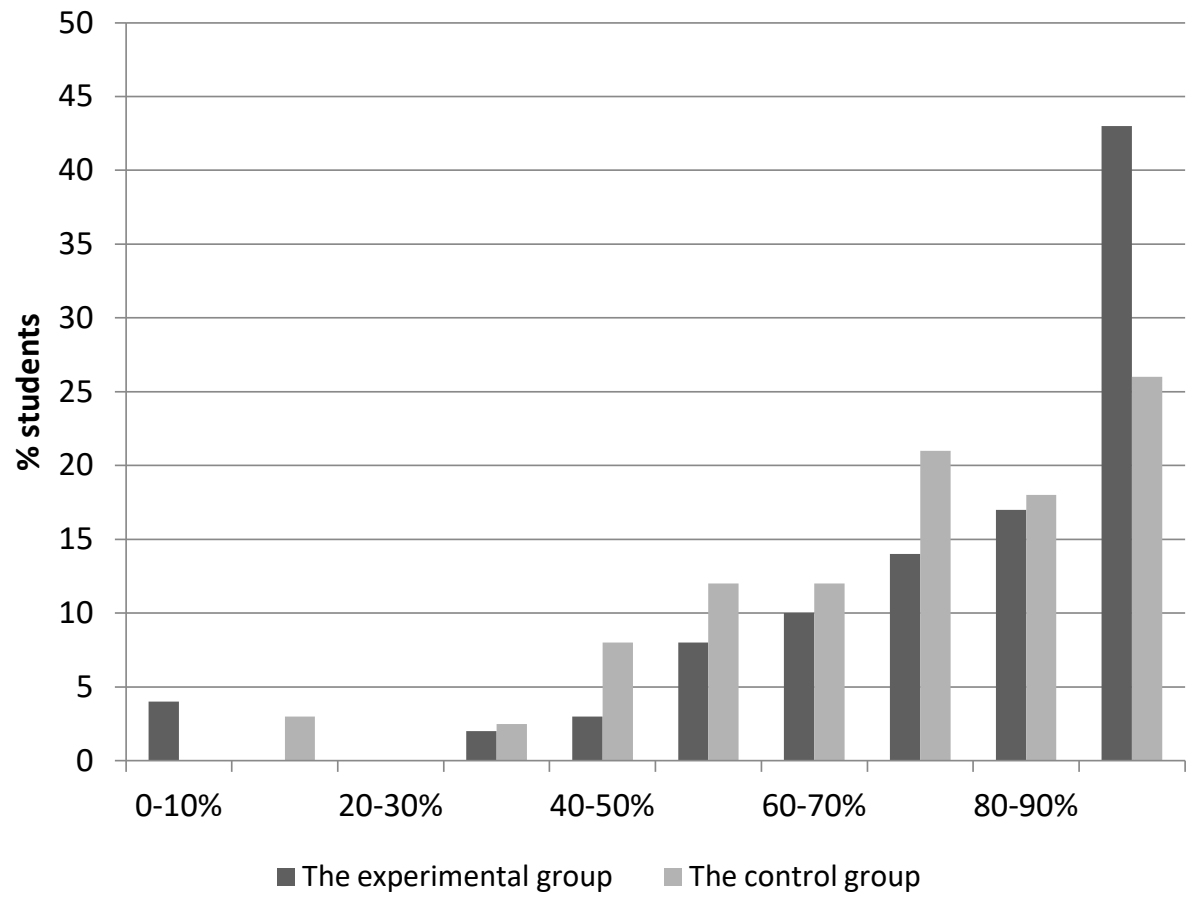

Fig. 5. The final control over the assimilation of AELC materials.

In addition, to assess the effectiveness of the proposed algorithms, a questionnaire was carried out for the students of the experimental group and demonstrated the positive reaction of the students to the introduction of AELC in the study of discrete mathematics.

Thus, when assessing adaptability to the level of assimilation of the material, $67.4 \%$ of respondents noted the option "high level of adaptability", $28.3 \%$ considered "the level of adaptability is rather high" and only $4.3 \%$ chose the option "I do not know".

The majority of respondents $(86.1 \%)$ noted an increase in motivation to study the discipline in AELC conditions, $13.8 \%$ abstained from an answer and the adaptation of the content seemed rather uninteresting only for $1.1 \%$ of respondents; no one spoke for the total lack of interest in the methods of adapting the educational content.

$67 \%$ of students noted that studying the discipline in AELC provides better mastery of the subject as compared to traditional teaching, 15\% noted the importance of the proposed approach and $18 \%$ found it difficult to answer this question.

Thus, the approbation of the adaptation algorithms proposed in this work within the framework of AELC in discrete mathematics showed their effectiveness in the educational process.

\section{Conclusions}

The adaptation algorithms of a mathematical educational content in the electronic environment are presented in the paper. A novelty of the proposed algorithms is to implement the content of the educational content in the three-level system: "introductory content adaptation" - "current content adaptation" - "appraisal-corrective adaptation." The proposed three-level system of adaptation of the mathematical educational content fulfills the functions of the student's "a conductor" from the beginning to the end of the training in 
the discipline, adapting the teaching materials taking into account the considered specificity of the formation of mathematical competence.

The implementation of the proposed adaptation algorithms to the mathematical content in AELC makes it possible to create individual educational trajectories for students within the framework of a flexible personalized educational schedule with multiple control of the process of self-education. When using the system presented in the work, for each student an optimal personal space of mathematical educational content is formed, which corresponds to its individual characteristics as much as possible.

The pedagogical experiment confirmed that the organization of the adaptation of the mathematical educational content allows the teacher to provide an individual approach to teaching students in the electronic environment, regardless of the number of students in the group, ensuring the simplicity of building a personal educational scenario for a student.

The results can also be used to develop AELC for other disciplines. Further development of the proposed adaptation algorithms of the educational content in a threelevel system is assumed by developing a formal model that includes control rules which correct the normative values of the content assimilation estimation based on the methods of expert assessments and fuzzy sets theory.

The reported study was funded by RFBR according to the research project №18-013-00654

\section{References}

1. Yu.V. Vainshtein, R.V. Esin, G.M. Tsibulsky, Education and Informatics, Adaptive model of developing individual educational trajectories for blended learning, 2, 86 (2017)

2. B. Lazarov, Information Models and Analyses, Application of some cybernetic models in building individual educational trajectory, 2, 90 (2013)

3. D.H. Clements, J. Sarama, Mathematical Thinking and Learning, Learning Trajectories in Mathematics Education, 6, 81 (2004)

4. V.V. Kureychik, V.V. Bova, Open Education, Modeling of Process Knowledge Representation in Intellectual Instructional Systems Based on Competence Approach, 3, 42 (2014)

5. G.A. Atanov, Educational Technology \& Society, The modeling of an educational subject domain or the subject model of a learner, 1, 111 (2001)

6. T. Murray, S. Blessing, S. Ainsworth, Interactive and Intelligent Educational Software, Springer: Netherlands, Authoring Tools for Advanced Technology Learning Environments: Toward Cost Effective Adaptive, 557 (2003)

7. O. Shabalina, S. Chickerur, Recent Patents on Computer Science, Technology centered higher education: best approaches and practices in technology integration, $\mathbf{9}$, 104 (2016)

8. E. Davidova, A. Sergushicheva, Open Education, Models of a student and a teacher for the multi-agent training system, 5, 25 (2015)

9. L. Zaytseva, E .Bul', Educational Technology \& Society, An adaptation in computer systems based on the structuring of learning objects, 9, 422 (2006)

10. M. Kolyada, Artificial Intelligence, The kinds of models trained in automated learning systems, 2, 28 (2008)

11. L. Rastrigin, Riga: Zinatne, An adaptive learning a the model of a learner, 160 (1988)

12. V. Wade, Digital Libraries and Archives: 13th Italian Research Conference on Digital Libraries, IRCDL 2017, Modena, Italy. An Adaptive Cross-Site User Modelling Platform for Cultural Heritage Websites, 733 (2017) 
13. E. O'Donnell, S. Lawless, M. Sharp, V. Wade, International Journal of Distance Education Technologies, A Review of Personalised E-Learning: Towards Supporting Learner Diversity, 13, 22 (2015)

14. O. Conlan, A. Staikopouslos, C. Hampson, S. Lawless, I. O'Keeffe, New Review of Hypermedia and Multimedia, The narrative approach to personalisation, 19, 132 (2013)

15. P. Brusilovsky, A. Kobas, W. Nejdl, Verlag Berlin Heidelberg. Springer, The adaptive web, methods and strategies of web personalisation (2007)

16. P. Brusilovsky, International Journal of Artificial Intelligence in Education, Adaptive and Intelligent Web-based Educational Systems, 13, 156 (2003)

17. W.J. Van der Linden, Springer Science, Business Media, LLC Statistical for social and behavioral sciences, Elements of adaptive testing, 437 (2010)

18. K. Hicks, Understanding The Top Learning Management Systems, URL: http://www.edudemic.com/the-20-best-learning-management-systems/ (2016)

19. Yu.V. Vainshtein, R.V. Esin, G.M. Tsibulsky, Bulletin of Krasnoyarsk State Pedagogical University, Adaptive e-learning resources for the advanced training of teaching staff, 2, 52 (2017)

20. V.A. Shershneva, L.V. Shkerina, V.N Sidorov, T.V. Sidorova, K.V. Safonov, European Journal of Contemporary Education, Contemporary Didactics in Higher Education in Russia, 17, 357 (2016) 